\title{
ARTíCULO \\ Variabilidad del coeficiente de absorción por fitoplancton con influencia de marea roja en las bahías de Manzanillo y Santiago, México
}

Variability of the absorption coefficient for phytoplankton influence of red tide in Manzanillo and Santiago Bays, Mexico

\author{
Ramón Sosa-Ávalos' ${ }^{1}$, Eduardo Millán-Núñez², Sonia I. \\ Quijano-Scheggia ${ }^{3}$, J. Rubén Lara-Lara ${ }^{4}$ y Lidia Silva-Iñiguez ${ }^{1}$
}

${ }^{1}$ Facultad de Ciencias Marinas, Universidad de Colima, Km. 20 carretera Manzanillo-Barra de Navidad, Manzanillo, México, 28860.rsosa@ucol.mx

${ }^{2}$ Departamento de Ecología Marina, División de Oceanología, Centro de Investigación Científica y Educación Superior de Ensenada, Carretera Ensenada-Tijuana No. 3918, Fracc. Zona Playitas, Ensenada, Baja California, México. C.P. 22860. emillan@cicese.mx.

${ }^{3}$ Centro Universitario de Investigaciones Oceanológicas, Universidad de Colima, Km. 20 carretera Manzanillo-Barra de Navidad, Manzanillo, México, 28860. quijano@ucol.mx.

${ }^{4}$ Departamento de Oceanografía Biológica, División de Oceanología, Centro de Investigación Científica y Educación Superior de Ensenada, Carretera Ensenada-Tijuana No. 3918, Fracc. Zona Playitas, Ensenada, Baja California, México, C.P. 22860. rlara@cicese.mx

\begin{abstract}
Five surveys (March, May, July, September and December 2004) in 6 stations at 3 depths (100, 10 and $\left.1 \% E_{0}(P A R)\right)$ were carried out in the bays of Manzanillo and Santiago, Mexico, to detect the temporal variation of water temperature, chlorophyll a concentration and specific absorption coefficient of phytoplankton at $440 \mathrm{~nm}$ and $676 \mathrm{~nm}\left(a_{\phi}^{*}(440), a_{\phi}^{*}(676)\right)$. The surface temperature was lower in spring and higher in summer. Meanwhile, the average surface chlorophyll a concentration changed from $1.1 \mathrm{mg} \mathrm{m}^{-3}$ in December to $20.8 \mathrm{mg} \mathrm{m}^{-3}$ in May, while integrated chlorophyll a in the euphotic zone was 36.2 to $146.6 \mathrm{mg} \mathrm{m}^{-2}$ in July and May, respectively. High chlorophyll a recorded in May was different from the other months as a result of harmful algal bloom event dominated by the dinoflagellate Ceratium balechii (Balech 1988) with an average abundance of $1.19 \times 10^{6}$ cells $L^{-1}$, which represent $95.31 \%$ of total phytoplankton. The average values of $a^{*}(440)$, $\mathrm{a}_{\phi}^{*}(676)$ were 0.051 and $0.029 \mathrm{~m}^{2}(\mathrm{mg} \mathrm{Chl} \mathrm{a})^{-1}$, respectively, which showed significant temporal variation between months, but had no differences through the euphotic zone. The spectra of the specific absorption coefficient of phytoplankton $\left(a_{\phi}^{*}(\lambda)\right)$ for May presented a clear packaging effect into the depths of 100 and $10 \% E_{0}(P A R)$. The ratio between the maximum blue/red spectral absorption $(440 \mathrm{~nm} / 676 \mathrm{~nm})$ showed lower values than 2.5 , indicating a phytoplankton community dominated primarily by microphytoplankton cells.
\end{abstract}

Key words: Specific absorption coefficient, chlorophyll a, harmful algal bloom, Manzanillo and Santiago Bays

Resumen.- Se realizaron 5 cruceros (marzo, mayo, julio, septiembre y diciembre 2004) en 6 estaciones a 3 profundidades $\left(100,10\right.$ y $1 \%$ de $\left.E_{0}(P A R)\right)$ en las bahías de Manzanillo y Santiago, México, para detectar la variación temporal de la temperatura, concentración de clorofila a y del coeficiente específico de absorción por fitoplancton a 440 y $676\left(a_{\phi}^{*}(440 \mathrm{~nm})\right.$, $\left.a_{\phi}^{*}(676 \mathrm{~nm})\right)$. La temperatura superficial fue menor en primavera y mayor en verano. La media de la concentración superficial de clorofila a cambió de $1,1 \mathrm{mg} \mathrm{m}^{-3}$ en diciembre a $20,8 \mathrm{mg} \mathrm{m}^{-3}$ en mayo, mientras que la clorofila a integrada en la zona eufótica fue de 36,2 a 146,6 $\mathrm{mg} \mathrm{m}^{-2}$ en julio y mayo, respectivamente. La alta clorofila a en mayo fue diferente a los otros meses como resultado de un florecimiento algal nocivo dominado por el dinoflagelado Ceratium balechii (Balech 1988) con una abundancia de $1,19 \times 10^{6}$ células $\mathrm{L}^{-1}$, el cual representa el 95,31\% del fitoplancton total. Los valores promedio del $\mathrm{a}_{\phi}^{*}(440)$ y $\mathrm{a}_{\phi}^{*}(676)$ fueron de 0,051 y $0,029 \mathrm{~m}^{2}(\mathrm{mg} \mathrm{Cla})^{-1}$, respectivamente, los cuales mostraron variación temporal significativa entre los meses, pero no tuvieron diferencias a través de la zona eufótica. Los espectros del coeficiente específico de absorción por fitoplancton $\left(a_{\phi}^{*}(\lambda)\right)$ presentaron en mayo un claro efecto de paquete a las profundidades del 100 y $10 \%$ de $E_{0}(P A R)$. La razón entre los máximos de absorción espectral azul/rojo $(440 \mathrm{~nm} / 676 \mathrm{~nm})$ mostraron valores menores a 2,5, indicando una comunidad de fitoplancton dominada principalmente por células del microfitoplancton.

Palabras clave: Coeficiente específico de absorción, clorofila a, florecimiento algal nocivo, bahías de Manzanillo y Santiago 


\section{INTRODUCCIÓN}

Los florecimientos algales nocivos (FAN) conocidos comúnmente como mareas rojas, se deben al aumento en la concentración de determinadas especies de microalgas, incluyendo dinoflagelados (Vargas-Montero \& Freer 2004). Estos fenómenos son el resultado de procesos físicos (surgencias de aguas más profundas, procesos de mezcla vertical) (Manrique \& Molina 1997), aporte de nutrientes por escurrimientos de agua en época de lluvias y contaminación marina (Kim et al. 2004), favoreciendo la acumulación de fitoplancton (Cortés-Altamirano \& PáezOsuna 1998). La alta concentración de microalgas durante un florecimiento suele causar la coloración del agua, convirtiéndola en rojo marrón o verde dependiendo de la especie predominante (Kahru \& Mitchell 1998, BarocioLeón et al. 2008). Se ha observado en cultivos microalgales que la velocidad de crecimiento no cambia, esto es específico de cada especie debido a la complejidad celular y talla. En la actualidad, estos eventos se están incrementando en las regiones costeras ocasionando problemas ambientales y de salud (Anderson 1995). Hasta ahora es escasa la información disponible entre las relaciones bio-ópticas in situ y las mareas rojas, esta asociación, aunado al monitoreo de los florecimientos producirán aportes importantes en el conocimiento de las propiedades bio-ópticas del fitoplancton (coeficiente de absorción de luz, dispersión, reflectancia, entre otras) (Kahru \& Mitchell 1998).

La determinación del coeficiente específico de absorción de luz por fitoplancton $\left(a_{\phi}^{*}(\lambda)\right)$ en aguas caso 2 (donde el color del océano se determina por las propiedades ópticas inherentes de la columna de agua como absorción y dispersión) es fundamental para entender el comportamiento fisiológico de la célula, y por lo tanto mejorar los modelos analíticos bio-ópticos en la interpretación del color del océano (IOCCG 2000). En los últimos años, se han estudiado ampliamente las curvas espectrales de absorción de luz en poblaciones de fitoplancton a diferentes escalas oceánicas (Babin et al. 2003), mostrando que el coeficiente específico $\left(a_{\phi}^{*}(\lambda)\right)$ varía de acuerdo con cambios en la composición celular de pigmentos y por el efecto de paquete debido al tamaño del fitoplancton. Durante la primavera de 2004 se presentó un evento de marea roja en las costas del Pacífico mexicano desde Puerto Vallarta hasta Lázaro Cárdenas, incluyendo las bahías de Manzanillo y Santiago, Colima. Por ello, el objetivo del presente estudio fue determinar la variabilidad temporal del $\left(a_{\phi}^{*}(\lambda)\right)$ y concentración de clorofila $a(\mathrm{Cl} a)$, haciendo énfasis en los datos recolectados durante el periodo de la marea roja. La concentración de clorofila $a$ y los coeficientes de absorción y específico de absorción de luz por fitoplancton fueron afectados por la marea roja que se presentó en mayo, ocasionando variación temporal de estas variables.

\section{MATERIALES Y MÉTODOS}

Las bahías de Manzanillo se encuentran localizadas al oeste del estado de Colima, en el litoral del Pacifico Mexicano (Fig. 1). Los muestreos se realizaron en 6 estaciones durante marzo, mayo, julio, septiembre y diciembre de 2004, obteniendo parámetros como el coeficiente de atenuación vertical de luz difusa $\left[K_{d}(\mathrm{PAR})\right]$ mediante la lectura de un disco de Secchi (LDS) y su relación empírica $K_{d}(\mathrm{PAR})=1,3 / \mathrm{LDS}($ Megard \& Berman 1989). Asimismo, se calcularon las profundidades de muestreo al 100, 10 y $1 \%$ de la irradiancia superficial $\left(E_{0}(P A R)\right)$ en cada una de las 6 estaciones de acuerdo con la ley de Lambert-Beer. La temperatura del agua se midió por medio de un sensor multiparámetros YSI-85.

\section{Clorofila $A$}

El agua de mar se recolectó por medio de una botella Niskin de 5 L de capacidad y se filtró un litro a través de filtros Whatman GF/F para determinar la concentración de la Cla. Los filtros fueron inmediatamente congelados en nitrógeno líquido hasta su análisis en el laboratorio. Los pigmentos fueron extraídos con acetona al $90 \%$ en frío y oscuridad por 24 h (Venrick \& Hayward 1984). El pigmento se determinó por medio de un espectrofotómetro Perkin Elmer lambda 35 (Parsons et al. 1984).

\section{COEFICIENTE ESPECÍFICO DE ABSORCIÓN DE LUZ POR FITOPLANCTON}

Las muestras de absorción por fitoplancton se colectaron en filtros de fibra de vidrio Whatman GF/F $(25 \mathrm{~mm}$ de diámetro), los cuales se almacenaron en un contenedor de nitrógeno líquido para su análisis en el laboratorio. Posteriormente, los filtros fueron descongelados e impregnados con agua de mar filtrada. Se analizaron en un espectrofotómetro con esfera integradora de luz (Shimadzu UV-2401 PC) siguiendo la guía de laboratorio descrita por Cleveland \& Weideman (1993). Se utilizó un barrido espectral entre 400-750 nm con resolución de 1 $\mathrm{nm}$, un ancho de banda espectral de $2 \mathrm{~nm}$ y una velocidad

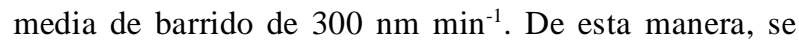
obtuvo la curva espectral de material particulado $\left(a_{p}(\lambda)\right)$, posteriormente los filtros se enjuagaron con metanol 


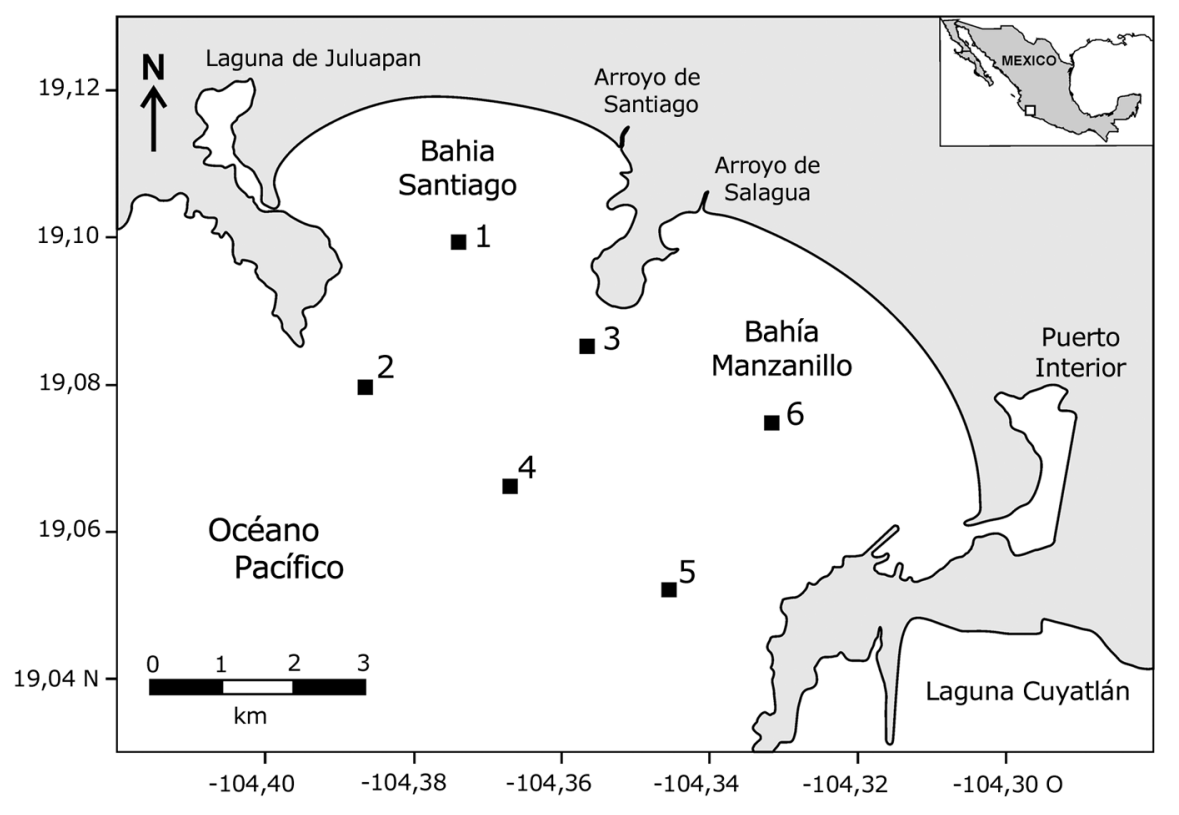

Figura 1. Localización de las estaciones de muestreo en las bahías de Manzanillo, México / Location of sampling stations in the bays of Manzanillo, Mexico

caliente durante un periodo de 30 min siguiendo la técnica de Kishino et al. (1985) para un segundo barrido espectral, teniendo como resultado la curva de detritus o material no pigmentado $\left(a_{d}(\lambda)\right.$. Con la diferencia entre el material particulado y el detritus, se obtuvo el coeficiente de absorción de luz por fitoplancton $\left(a_{\phi}(\lambda)\right)$, según la ecuación 1:

$$
a_{\phi}(\lambda)=a_{p}(\lambda)-a_{d}(\lambda)
$$

donde $a_{p}(\lambda)$ es la absorción de luz por material particulado a cierta longitud de onda $\left(\mathrm{m}^{-1}\right), a_{\phi}(\lambda)$ es el coeficiente de absorción de luz por fitoplancton $\left(\mathrm{m}^{-1}\right), \mathrm{y} a_{d}(\lambda)$ absorción de luz por detritus $\left(\mathrm{m}^{-1}\right)$. El coeficiente específico de absorción de luz por fitoplancton $\left(a_{\phi}^{*}(\lambda)\left(\mathrm{m}^{2}(\mathrm{mg} \mathrm{Cl} a)^{-1}\right)\right.$ se obtuvo normalizando la absorción $\left(\mathrm{m}^{-1}\right)$ por la concentración de clorofila $a\left(\mathrm{mg} \mathrm{m}^{-3}\right)$. Los coeficientes de absorción se corrigieron por un factor que ajusta la densidad óptica de las muestras en filtros $\left(\mathrm{OD}_{\mathrm{s}}(\lambda)\right.$ y lo relaciona con la densidad óptica en muestras en suspensión $\left(\mathrm{OD}_{\mathrm{s}}(\lambda)\right.$ (ecuación 2 , que es tomada de referencia).

$$
\mathrm{OD}_{\mathrm{S}}=0,3269 \mathrm{OD}_{\mathrm{f}}+0,4773\left(\mathrm{OD}_{\mathrm{f}}\right)^{2}
$$

\section{ANÁLISIS DEL FITOPLANCTON}

Las muestras de agua fueron recolectadas con botella Niskin de $5 \mathrm{~L}$ de capacidad de cada profundidad (100, 10 y $1 \%$ de $E_{0}(P A R)$, de las cuales se recolectaron $500 \mathrm{ml}$ de muestra y se fijaron con solución lugol para determinar la abundancia del fitoplancton, en total se analizaron 18 muestras y solamente se colectaron muestras durante el evento de marea roja. La cuantificación del fitoplancton se realizó colocando $10 \mathrm{ml}$ de muestra en cámaras de sedimentación por $24 \mathrm{~h}$, utilizando un microscopio invertido de contraste de fase Carl Zeiss (con cámara incluida) en 400x de acuerdo al método descrito por Utermöhl (1958). Se utilizaron claves especializadas para la identificación del fitoplancton y en particular el Ceratium balechii se identificó de acuerdo a Meave del Castillo et al. (2003) y Gárate-Lizárraga (2009).

El análisis estadístico de los datos se realizó por Statistica StatSoft ver.7 utilizando Kruskal-Wallis o ANDEVA de una vía para determinar si existían diferencias significativas entre los meses, y para conocer cuales meses fueron diferentes se utilizó una comparación múltiple por rangos no paramétrica. Asimismo, los datos fueron analizados por el método de ANDEVA factorial para conocer su comportamiento espacial entre las 
estaciones y las profundidades de muestreo, todos los análisis fueron realizados con un nivel de significancia del 5\% (Zar 1999).

\section{Resultados}

El promedio más bajo de temperatura superficial del mar en el área de estudio se presentó en marzo y mayo $(27,30$ y $27,45^{\circ} \mathrm{C}$, respectivamente) y el más alto en septiembre de $30,38^{\circ} \mathrm{C}$ (Tabla 1 ), esto permitió encontrar una variación temporal $(P=0,0001)$ entre la temperatura de julio y septiembre con la de diciembre, marzo y mayo. La distribución vertical de la temperatura mostró una columna de agua homogénea durante verano (julio y septiembre), esto debido a los procesos de mezcla vertical, mientras que en diciembre se encontró un gradiente de temperatura entre el 10 y $1 \%$ de la $E_{0}(P A R)$ (Fig. 2), en marzo y mayo no se observó con claridad el gradiente de temperatura (Figs. $2 \mathrm{a}$, c y f). Sin embargo, en las estaciones 2,4 y 5 presentaron cambios significativos de temperatura (Figs. 2b, d y e). En general, la temperatura mostró variabilidad espacial en la zona eufótica $(P<0,05)$ y homogeneidad entre las estaciones.

Tabla 1. Valores promedio de la temperatura superficial $\left(\mathrm{T}^{\circ} \mathrm{C}\right)$, clorofila a $\left(\mathrm{Cla}, \mathrm{mg} \mathrm{m}^{-3}\right)$, clorofila a integrada $\left(\mathrm{Cla}, \mathrm{mg} \mathrm{m}^{-2}\right)$, profundidad de la zona eufótica (Zeu, $m)$, coeficiente de absorción de luz $\left(a_{\phi}, m^{-1}\right)$, razón de absorción $\left(a_{\phi}^{*}(440): a_{\phi}^{*}(676)\right), y$ coeficiente específico de absorción de luz por fitoplancton $\left[a_{\phi}^{*}, \mathrm{~m}^{2}(\mathrm{mg} \mathrm{Cla})^{-1}\right]$ a 440 y $676 \mathrm{~nm}$ en las bahías de Manzanillo y Santiago. Average values of Surface temperature $\left(\mathrm{T}^{\circ} \mathrm{C}\right)$, chlorophyll a $\left(\mathrm{Cla}, \mathrm{mg} \mathrm{m}^{-3}\right)$, integrated chlorophyll a $\left(\mathrm{Cla}, \mathrm{mg} \mathrm{m}^{-2}\right)$, depth of the euphotic zone (Zeu, $m$ ), light absorption coefficient $\left(a_{\phi^{\prime}} \mathrm{m}^{-1}\right)$, absorption ratio $\left(a_{\phi}^{*}(440): a_{\phi}^{*}(676)\right)$, and phytoplankton specific light absorption coefficient $\left[\mathrm{a}_{\phi^{*}}^{*} \mathrm{~m}^{2}(\mathrm{mg} \mathrm{Cla})^{-1}\right]$ at 440 and $676 \mathrm{~nm}$ in the Manzanillo and Santiago Bays

\begin{tabular}{cccccc}
\hline & \multicolumn{4}{c}{ A ñ o 2004} \\
Variables & Marzo & Mayo & Julio & Septiembre & Diciembre \\
\cline { 2 - 5 } & 27,30 & 27,45 & 30,03 & 30,38 & 28,05 \\
Tla $(100 \%)$ & 1,2 & 20,8 & 1,2 & 1,7 & 1,1 \\
Cla $(10 \%)$ & 1,6 & 10,0 & 1,0 & 1,8 & 1,4 \\
Cla $(1 \%)$ & 0,7 & 2,0 & 1,4 & 1,4 & 1,1 \\
Cla int & 38,1 & 146,6 & 36,2 & 46,1 & 47,2 \\
$a_{\phi}(440) 100 \%$ & 0,091 & 0,219 & 0,051 & 0,037 & 0,054 \\
$a_{\phi}(676) 100 \%$ & 0,049 & 0,124 & 0,032 & 0,023 & 0,023 \\
$a_{\phi}^{*}(440) 100 \%$ & 0,092 & 0,012 & 0,062 & 0,031 & 0,057 \\
$a_{\phi}^{*}(676) 100 \%$ & 0,048 & 0,007 & 0,033 & 0,019 & 0,026 \\
$a_{\phi}(440) 10 \%$ & 0,099 & 0,147 & 0,040 & 0,047 & 0,098 \\
$a_{\phi}(676) 10 \%$ & 0,058 & 0,085 & 0,030 & 0,030 & 0,041 \\
$a_{\phi}^{*}(440) 10 \%$ & 0,066 & 0,020 & 0,040 & 0,028 & 0,069 \\
$a_{\phi}^{*}(676) 10 \%$ & 0,039 & 0,012 & 0,028 & 0,018 & 0,028 \\
$a_{\phi}(440) 1 \%$ & 0,064 & 0,065 & 0,063 & 0,072 & 0,031 \\
$a_{\phi}(676) 1 \%$ & 0,036 & 0,038 & 0,042 & 0,043 & 0,022 \\
$a_{\phi}^{*}(440) 1 \%$ & 0,107 & 0,041 & 0,046 & 0,061 & 0,025 \\
$a_{\phi}^{*}(676) 1 \%$ & 0,060 & 0,024 & 0,031 & 0,036 & 0,017 \\
$a_{\phi}^{*}(440): a_{\phi}^{*}(676) 100 \%$ & 1,93 & 1,73 & 1,87 & 1,62 & 2,25 \\
$a_{\phi}^{*}(440): a_{\phi}^{*}(676) 10 \%$ & 1,70 & 1,76 & 1,42 & 1,61 & 2,45 \\
$a_{\phi}^{*}(440): a_{\phi}^{*}(676) 1 \%$ & 1,79 & 1,73 & 1,48 & 1,70 & 1,43 \\
Zeu (m) $1 \%$ & 31,0 & 15,0 & 37,0 & 28,0 & 39,0 \\
\hline
\end{tabular}


Temperatura $\left({ }^{\circ} \mathrm{C}\right)$
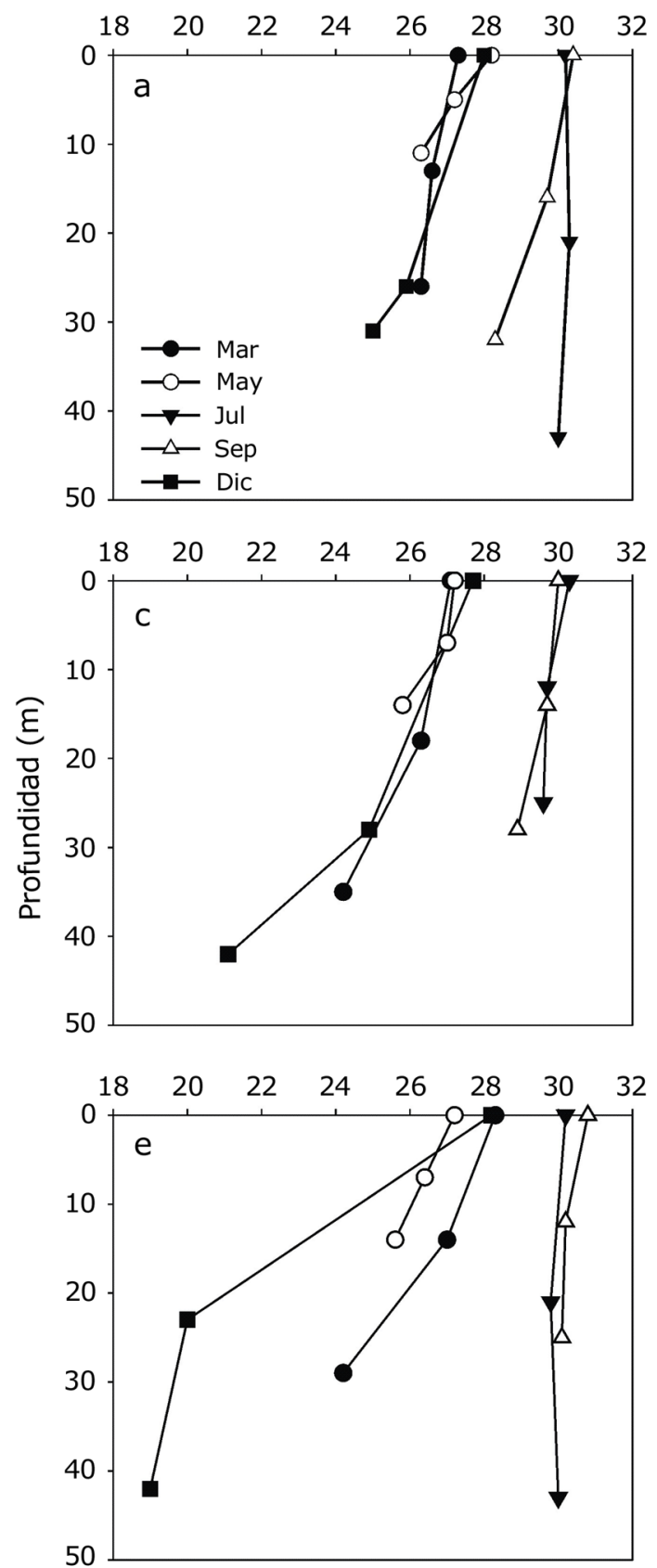

Temperatura $\left({ }^{\circ} \mathrm{C}\right)$
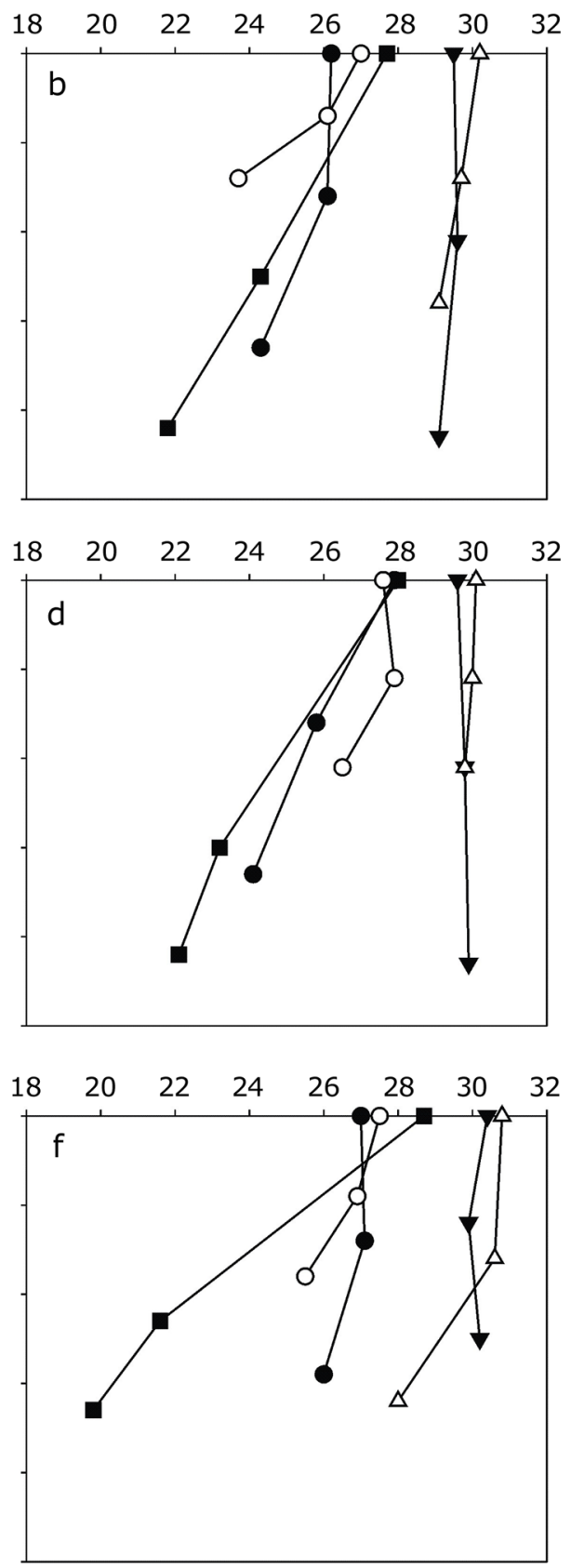

Figura 2. Distribución vertical de temperatura in situ $\left(T^{\circ} \mathrm{C}\right)$ durante los cruceros realizados en bahías de Manzanillo. a) E-1, b) E-2, c) E-3, d) E-4, e) E-5 y f) E-6 / Vertical distribution of in situ temperature $\left(T^{\circ} \mathrm{C}\right)$ during the cruises in Manzanillo Bays. a) E-1, b) E-2, c) E-3, d) E-4, e) E-5 and f) E-6 

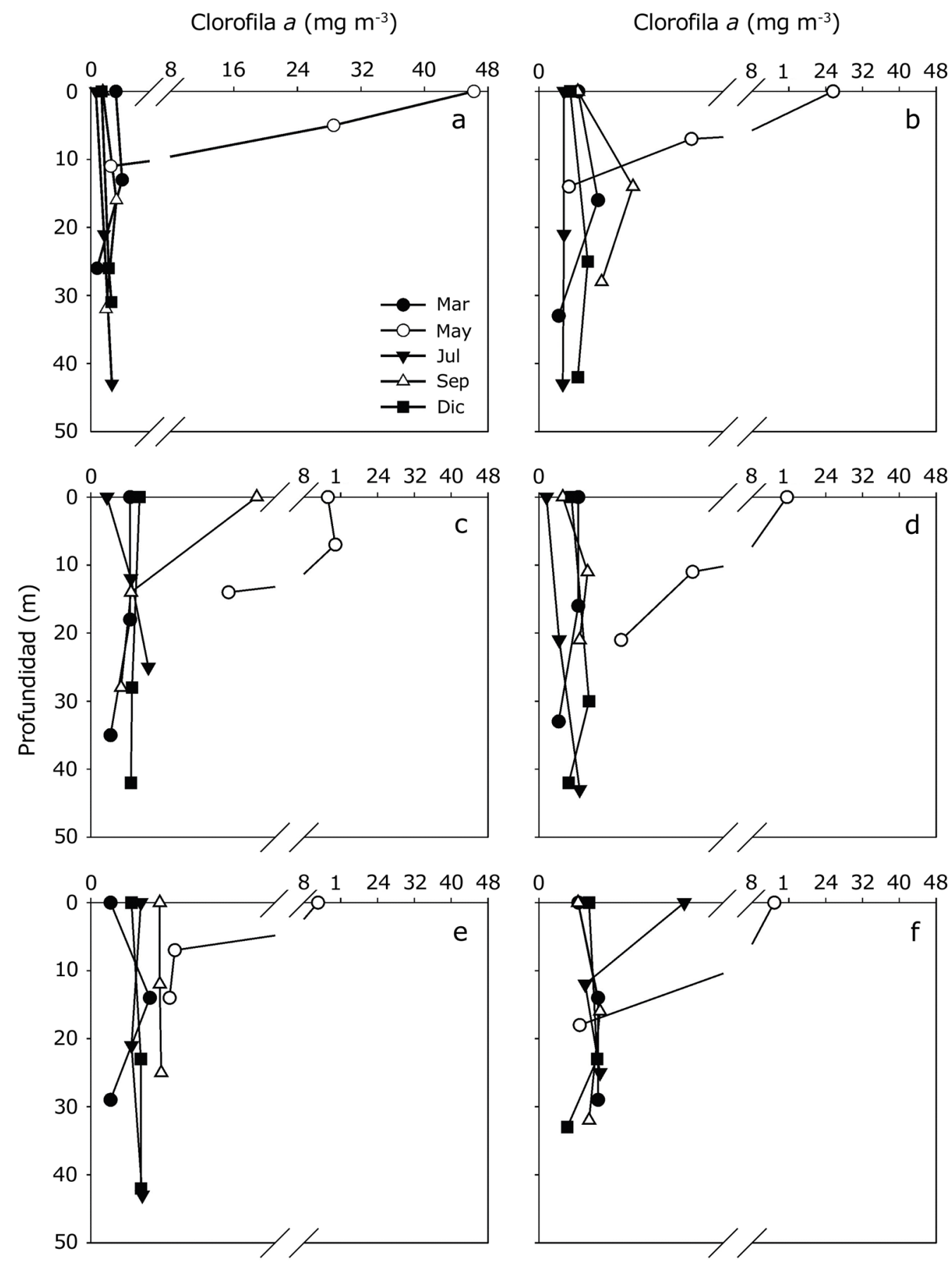

Figura 3. Distribución vertical de la concentración de clorofila a durante los cruceros en bahías de Manzanillo. a) E-1, b) E-2, c) E-3, d) E-4, e) E-5 y f) E-6. El eje de las $X$ se interrumpe entre 5 a $8 \mathrm{mg} \mathrm{m}^{-3} /$ Vertical distribution of the chlorophyll a concentration during the cruises in Manzanillo Bays. a) E-1, b) E-2, c) E-3, d) E-4, e) E-5 and f) E-6. The $X$ axis is interrupted between 5 to $8 \mathrm{mg} \mathrm{m}^{-3}$ 
La concentración promedio superficial de la clorofila $a$ mostró cambios desde $1,1 \mathrm{mg} \mathrm{m}^{-3}$ en diciembre hasta 20,8 $\mathrm{mg} \mathrm{m}^{-3}$ en mayo (Tabla 1), esto como resultado de un intenso evento de florecimiento de fitoplancton que tuvo una duración mayor a un mes. En la profundidad del $10 \%$ de $E_{0}(P A R)$ la Cla mostró un intervalo entre 1,0 y $10 \mathrm{mg} \mathrm{m}^{-3}$ durante julio y mayo, respectivamente, mientras que al $1 \%$ el promedio cambió entre $0,7 \mathrm{mg} \mathrm{m}^{-3}$ en marzo y $\sim 2,0$ $\mathrm{mg} \mathrm{m}^{-3}$ en mayo (Tabla 1). La clorofila integrada también presentó valores altos durante mayo hasta de 146,6 mg $\mathrm{m}^{-2}$, debido al efecto de la marea roja y valores bajos 36,2 $\mathrm{mg} \mathrm{m}^{-2}$ para julio. La alta concentración de $\mathrm{Cl} a$ registrada en mayo fue significativamente diferente a la de los otros meses $(P=0,0001)$. En general, el perfil vertical de la $\mathrm{Cl} a$ presentó homogeneidad entre las estaciones y en su gran mayoría el valor máximo se observó a la profundidad del $10 \%$ de $E_{0}(P A R)$ durante julio y diciembre (Fig. 3).

Se identificaron en total 6 especies de fitoplancton durante el evento de florecimiento algal que se presentó en mayo de 2004. Las especies con mayor densidad celular fueron Ceratium balechii (Fig. 4) con 1,19 x $10^{6}$ células $\mathrm{L}^{-1}$ que representó el $95,31 \%$ de la abundancia total y Prorocentrum micans con $3,88 \times 10^{4}$ células $\mathrm{L}^{-1}$ que significó el 3,11\% del fitoplancton total (Tabla 2).

Tabla 2. Taxonomía del fitoplancton identificado en el florecimiento algal de mayo 2004 en las bahías de Manzanillo y Santiago / Taxonomy of phytoplankton algal bloom identified in May 2004 in the bays of Manzanillo and Santiago

\begin{tabular}{lcc}
\hline \multicolumn{1}{c}{ Especie } & $\begin{array}{c}\text { Abundancia celular } \\
\left(\text { células L }{ }^{-1} \text { ) }\right.\end{array}$ & Porcentaje \\
\hline Ceratium balechii & 1190000 & 95,31 \\
Ceratium furca & 1130 & 0,090 \\
Dinophysis sp. & 16000 & 1,28 \\
Lingulodinium polyedrum & 1600 & 0,13 \\
Peridinium sp. & 1000 & 0,08 \\
Prorocentrum micans & 38800 & 3,11 \\
Total & 1250000 & \\
\hline
\end{tabular}

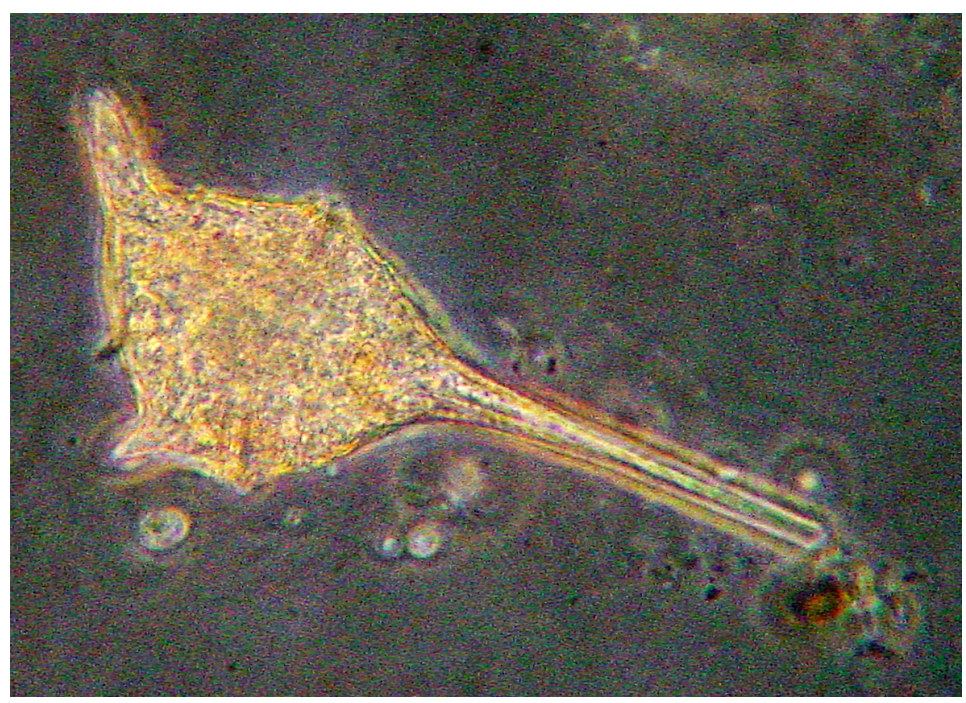

Figura 4. Microfotografía del dinoflagelado Ceratium balechii que fue la especie dominante durante el florecimiento algal nocivo de mayo 2004 en las bahías de Manzanillo y Santiago / Microphotograph of the dinoflagellate Ceratium balechii was the dominant specie harmful algal bloom during May 2004 in the Manzanillo and Santiago Bays 
Los hombros de la curva espectral donde los pigmentos del fitoplancton absorben mayormente la luz son en la banda azul $(440 \mathrm{~nm})$ y roja $(676 \mathrm{~nm})$. En este estudio, el mayor coeficiente de absorción se presentó a la longitud de onda de $440 \mathrm{~nm}\left(a_{\phi}(440)\right)$ con promedio en la superficie de 0,037 a $0,219 \mathrm{~m}^{-1}$ en septiembre y mayo, respectivamente (Tabla 1). A la profundidad del $10 \%$ de $E_{0}(P A R)$ el promedio varió de $0,040 \mathrm{~m}^{-1}$ en julio a $0,147 \mathrm{~m}^{-1}$ en mayo, mientras que al $1 \%$ los valores estuvieron entre 0,031 a $0,072 \mathrm{~m}^{-1}$ en diciembre y septiembre, respectivamente. Por otro lado, el promedio en la superficie del $a_{\phi}$ (676) mantuvo el valor de $0,023 \mathrm{~m}^{-1}$ durante septiembre y diciembre, llegando a un máximo de $0,124 \mathrm{~m}^{-1}$ en mayo, a diferencia del $10 \%$ de $E_{0}(P A R)$ que mostró valores de 0,030 a $0,085 \mathrm{~m}^{-1}$ en julio y mayo, respectivamente; mientras que al $1 \%$ se registró un intervalo de 0,022 a $0,043 \mathrm{~m}^{-1}$ durante diciembre y septiembre, respectivamente (Tabla 1). En general, la mayor magnitud del coeficiente $a_{\phi}(\lambda)$ se presentó al $100 \%$ y $10 \%$ de $E_{0}(P A R)$ en mayo, alcanzando valores hasta de $0,32 \mathrm{~m}^{-1}$ (Fig. 5a, 5b). Los máximos valores de $a_{\phi}(\lambda)$ estuvieron asociados con la alta concentración de clorofila $a$ como resultado de la marea roja que se presentó en ese periodo.

En mayo los valores del coeficiente específico $a_{\phi}^{*}(440)$ mostró un promedio en superficie de $0,012 \mathrm{~m}^{2}(\mathrm{mg} \mathrm{Cla})^{-1} \mathrm{a}$ $0,092 \mathrm{~m}^{2}(\mathrm{mg} \mathrm{Cl} a)^{-1}$ durante marzo. En la profundidad del $10 \%$ de $E_{0}(P A R)$ los valores fueron desde 0,020 a $0,069 \mathrm{~m}^{2}$ $(\mathrm{mg} \mathrm{Cl} a)^{-1}$ entre mayo y diciembre (Tabla 1$)$. Por su parte el $a_{\phi}^{*}(676)$ al nivel de $100 \%$ de luz registró un promedio de $0,007 \mathrm{~m}^{2}(\mathrm{mg} \mathrm{Cl} a)^{-1}$ en mayo, y valores altos de $0,048 \mathrm{~m}^{2}$ $(\mathrm{mg} \mathrm{Cl} a)^{-1}$ en marzo; por otro lado, el promedio de mayo a $676 \mathrm{~nm}$ al $10 \%$ fue de $0,012 \mathrm{~m}^{2}\left(\mathrm{mg} \mathrm{Cla}^{-1}\right.$ (Tabla 1). En general, los valores de $a_{\phi}^{*}(440)$ y $a_{\phi}^{*}(676)$ mostraron una variabilidad temporal significativa $(P<0,05)$ entre los meses de muestreo (Fig. 6).

La razón de absorción promedio entre las bandas espectrales $\left(a_{\phi}^{*}(440) / a_{\phi}^{*}(676)\right)$ tuvo valores en superficie de 1,62 a 2,25 entre septiembre y diciembre, a diferencia de la profundidad del $10 \%$ de $E_{0}(P A R)$ que mostró un intervalo entre 1,42 en julio y 2,45 en diciembre; mientras que al $1 \%$ la razón espectral fue de 1,43 en diciembre a 1,79 en marzo (Tabla 1). En general, se puede asumir que la razón de absorción en este estudio presentó valores bajos, indicando un tamaño de células relacionadas principalmente con el microfitoplancton.
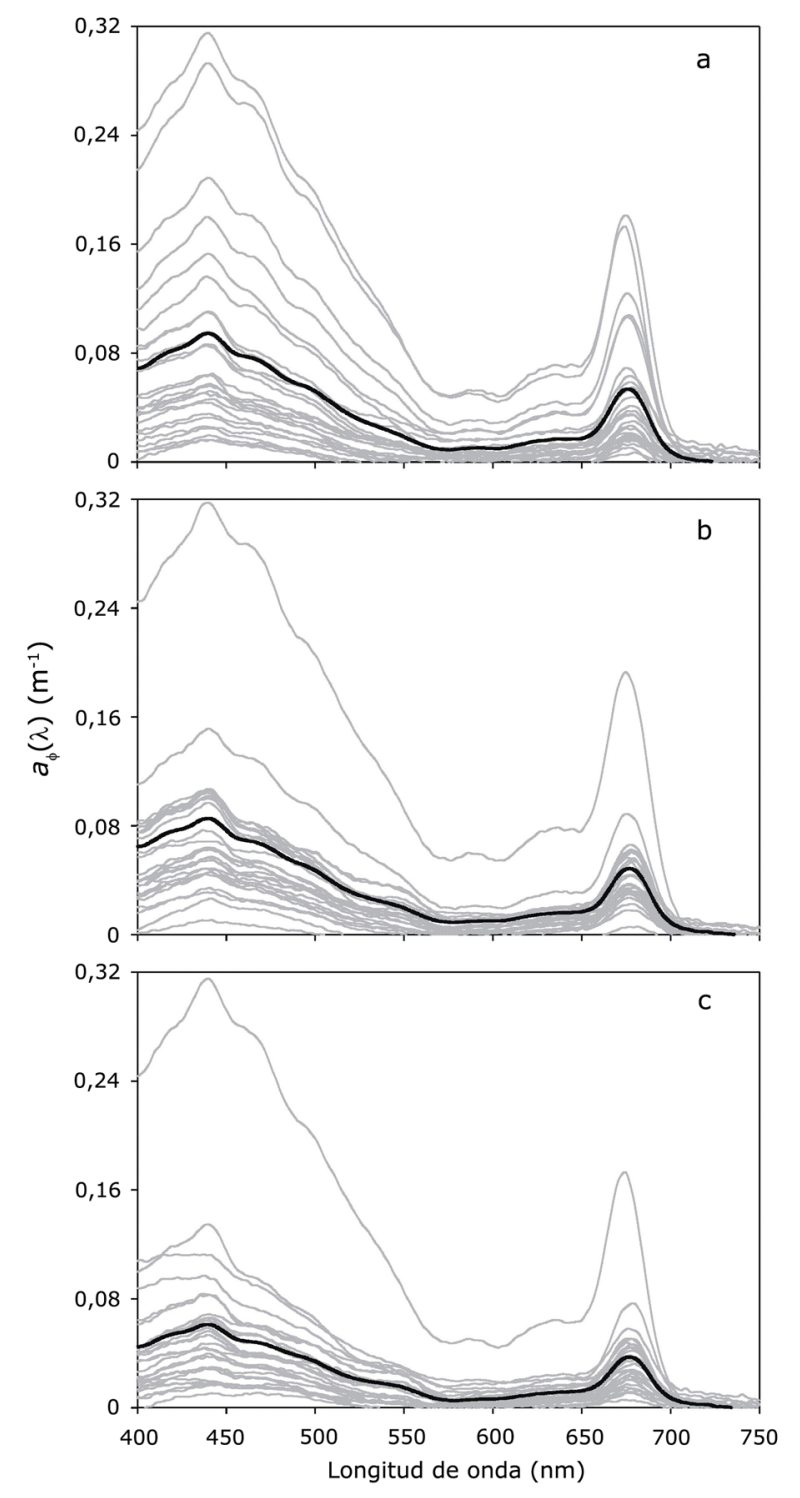

Figura 5. Espectros del coeficiente de absorción de luz por fitoplancton $\left(a_{\phi}(\lambda)\right)$ a través de la zona eufótica en bahías de Manzanillo. Las líneas delgadas representan cada uno de los espectros de $\left(a_{\phi}(\lambda)\right)$ y la línea gruesa el promedio. a) $100 \%$, b) $10 \%$ y c) $1 \% E_{0}$ (PAR) / Spectra of light absorption coefficient for phytoplankton $\left(a_{\phi}(\lambda)\right)$ through the euphotic zone in Manzanillo Bays. The thin lines represent each $\left(a_{\phi}(\lambda)\right)$ spectra and the thick line represent the average. a) $100 \%$, b) $10 \%$ and c) $1 \% E_{0}(P A R)$ 

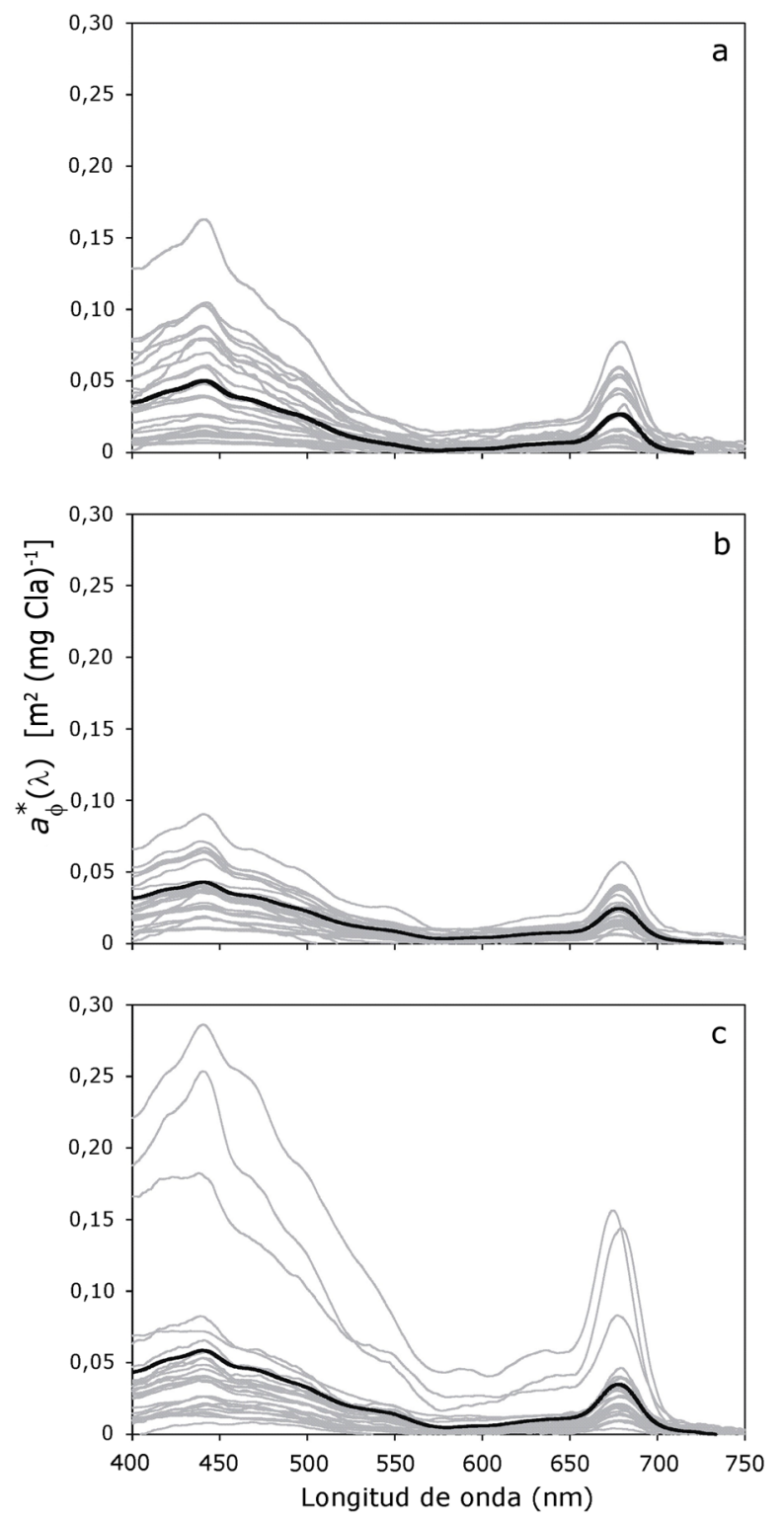

Figura 6. Coeficiente específico de absorción de luz por fitoplancton $\left(a^{*}(\lambda)\right)$ a través de la zona eufótica en bahías de Manzanillo. Las líneas delgadas representan cada uno de los espectros de $\left(a_{\phi}^{*}(\lambda)\right)$ y la línea gruesa el promedio. a) $100 \%$, b) $10 \%$ y c) $1 \% \mathrm{E}_{0}$ (PAR) / Specific light absorption coefficient for phytoplankton $\left(a_{\phi}^{*}(\lambda)\right)$ through the euphotic zone in Manzanillo Bays. The thin lines represent each spectral of $\left(a_{\phi}^{*}(\lambda)\right)$ and the thick line represent the average. a) $100 \%$, b) $10 \%$ and c) $1 \% \mathrm{E}_{0}(\mathrm{PAR})$
Los valores de $a_{\phi}(440)$ y $a_{\phi}(676)$ de todos los meses se agruparon y se ajustaron a una función de potencia con respecto a la concentración de clorofila $a$, las diferentes curvas mostraron un coeficiente de determinación $\left(\mathrm{r}^{2}\right)$ de 0,75 y 0,74 , respectivamente, indicando que la clorofila $a$ explicó el 75 y $74 \%$ de la variabilidad de los coeficientes de absorción (Figs. 7a y b). Sin embargo, cuando se ajustaron los datos de $a_{\phi}(440)$ y $a_{\phi}(676)$ de mayo (evento de marea roja), la clorofila $a$ explicó hasta un $89 \%$ de su variabilidad (Figs. 7c y d).

\section{Discusión}

La región noreste del océano Pacífico tropical $\left(12^{\circ} \mathrm{N}\right.$ y $\left.23^{\circ} \mathrm{N}\right)$ es una zona de transición tropical-subtropical influenciada significativamente por procesos oceanográficos advectivos de gran escala, los cuales son afectados por la Corriente de California y por la Subsuperficial Subtropical (Godínez et al. 2010, Kurczyn et al. 2012). En general, los datos de temperatura mostraron un comportamiento estacional con valores altos en verano y bajos durante invierno y primavera. Esta variación temporal es el resultado del efecto de la Corriente de California con agua fría durante los meses de invierno y primavera, y por la Corriente Costera Mexicana (CCM) con agua cálida durante junio a noviembre ocasionando un desplazamiento hacia el norte de Cabo Corrientes (Badán-Dangón 1997).

La alta abundancia de fitoplancton durante mayo estuvo asociado al evento de marea roja dominada por el dinoflagelado Ceratium balechii. Meave del Castillo et al. (2003) encontraron a esta especie ampliamente distribuida en el Pacífico tropical mexicano desde el Golfo de California hasta Oaxaca, y es responsable de la formación de continuos florecimientos algales en la región. En mayo la biomasa de la clorofila en superficie representó $\sim 95 \%$, mientras que la integrada en la zona eufótica fue de $\sim 75 \%$. El análisis estadístico de Kruskal-Wallis mostró una clara variación temporal con la clorofila del mes de mayo en relación al resto de los meses. Por otro lado, Barocio-León et al. (2008) mostraron evidencias de marea roja frente a Baja California con una concentración de $\mathrm{Cl} a$ de $\sim 8,60 \mathrm{mg}$ $\mathrm{m}^{-3}$ en la superficie. Estos valores fueron un $50 \%$ menor a la Cla observada en la superficie durante el presente trabajo; de igual manera, los datos de Castro-Ochoa (2010) reportaron valores promedios de Cla entre 4,45 y 1,00 mg $\mathrm{m}^{-3}$ durante verano en la bahía de Santiago, valores que fueron un 59 y $30 \%$ menor a los presentados en este trabajo; se considera que estas diferencias se debieron principalmente al efecto de la marea roja del 2004. 

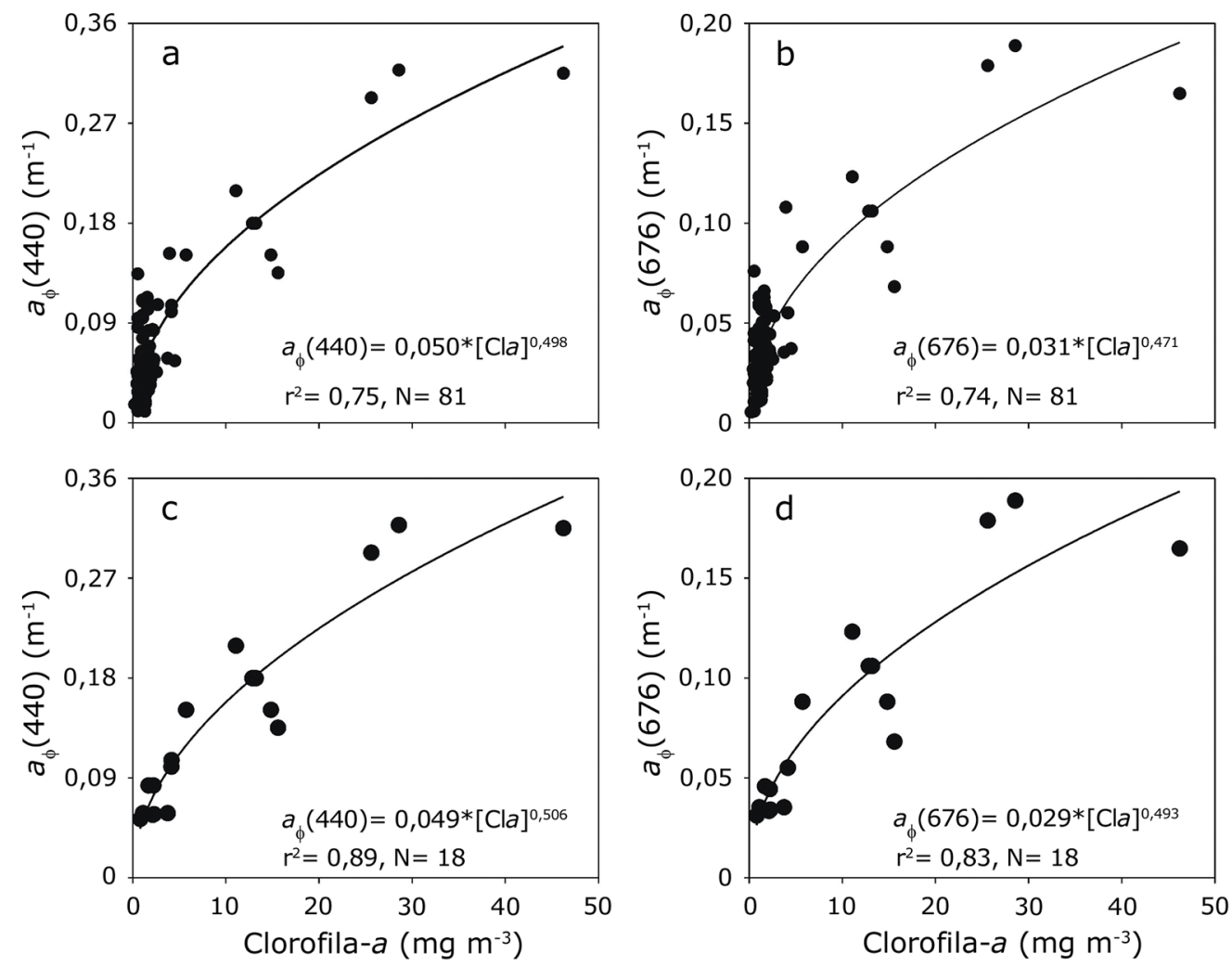

Figura 7. Variación del coeficiente de absorción de luz por fitoplancton a 440 y $676 \mathrm{~nm}$ como una función de la concentración de clorofila a. La línea continua representa el ajuste de todos los datos a una función de potencia (a, b). Ajuste a los datos observados en mayo durante la marea roja (c, d) / Variation of the light absorption coefficient by phytoplankton at 440 and $676 \mathrm{~nm}$ as a function of the chlorophyll a concentration. The solid line represents the fit of all data to a power function $(a, b)$. Fit to the observed data in May during the red tide (c, d)

Los análisis del $a_{\phi}^{*}(440 \mathrm{~nm})$ en este estudio, permiten entender la fisiología del fitoplancton y la óptica hidrológica de las aguas costeras de las bahías de Manzanillo, así como a mejorar en un futuro cercano los modelos bio-ópticos analíticos regionales para la interpretación del color del océano (Bricaud et al. 2004). Estos modelos funcionan adecuadamente cuando se aplican correctamente en relación a un cuerpo de agua como el Caso 2 para este estudio, donde el color del océano se determina por las propiedades ópticas inherentes de la columna de agua, así como por eventos extraordinarios como el desarrollo de mareas rojas que también contribuyen a la variabilidad óptica hidrológica (Sathyendranath et al. 2000). En general, en este estudio los datos del coeficiente específico durante marzo fueron $87 \%$ mayor que en mayo en superficie, así como un $71 \%$ más alto en diciembre que mayo en relación al $10 \%$ de $E_{0}(P A R)$; por lo tanto es posible que los valores bajos del coeficiente específico que se observaron en mayo fueron como resultado del intenso evento de marea roja que incrementó la concentración de clorofila $a$ en superficie y al $10 \%$ de $E_{0}(P A R)$, ocasionando un efecto de paquete por el tamaño dominante de células del microfitoplancton. En promedio, los coeficientes específico $a_{\phi}^{*}(440)$ y $a_{\phi}^{*}(676)$ fueron de 0,051 y $0,029 \mathrm{~m}^{2}(\mathrm{mg} \mathrm{Cla})^{-1}$, respectivamente, a diferencia de cuando se presentó la marea roja que fueron de 0,025 y $0,014 \mathrm{~m}^{2}(\mathrm{mg} \mathrm{Cla})^{-1}$, estos valores fueron menores en magnitud a los reportados por Barocio-León et al. (2008) y a los de Sosik \& Mitchell (1995) para el Sistema de la Corriente de California. Por otro lado, Sosa-Ávalos et al. (2010) reportaron un valor promedio de $0,048 \mathrm{~m}^{2}(\mathrm{mg} \mathrm{Cla})$ ${ }^{1}$ durante 4 cruceros estacionales en 1999 frente a Baja California, con valores muy similares a los presentados en este estudio para las bahías de Manzanillo. Los autores anteriormente mencionados, reportaron una variabilidad espacial para $a_{\phi}^{*}(440)$ y $a_{\phi}^{*}(676)$ en superficie, 10 y $1 \%$ de 
$E_{0}(P A R)$ durante primavera y otoño, a diferencia de lo observado en las bahías de Manzanillo que no se detectó variación con la profundidad. Esto posiblemente se debió a que las muestras colectadas frente a Baja California estuvieron alejadas de la costa a una distancia de $20 \mathrm{mn}$ y a profundidades mayores de $\mathrm{Z}_{\text {eu }}$; mientras que en las bahías de Manzanillo las estaciones fueron costeras y la profundidad de la zona eufótica fue somera (Tabla 1). Asimismo, Millán-Núñez et al. (2004) y Millán-Núñez \& Millán-Núñez (2010) determinaron frente a la península de Baja California, México, en enero de 2001 y 2002 valores de $a_{\phi}^{*}(440)$ y $a_{\phi}^{*}(676)$ similares a los reportados en el presente estudio.

De acuerdo a los valores de la razón de absorción entre las bandas espectrales $\left(a_{\phi}^{*}(440) / a_{\phi}^{*}(676)\right)$, se observaron en este estudio valores menores a 2,5 , lo que sugiere que la comunidad del fitoplancton estuvo dominada por células entre 20-200 $\mu \mathrm{m}$ de tamaño, tal y como lo reporta Wu et al. (2007) para la región sur de China donde detectaron valores menores de 3,0 durante un florecimiento de diatomeas. Asimismo, estos resultados concuerdan con el trabajo de Barocio-León et al. (2008) donde reportaron valores menores a 2,0 en altas abundancias de células del microfitoplancton. Kahru \& Mitchell (1998) observaron una disminución de los valores de absorción en los espectros durante un evento de marea roja al sur de California, comportamiento muy similar a los reportados en este estudio; por lo que consideramos que la disminución de la magnitud de los espectros se debió principalmente al efecto de paquete que se produce por una alta concentración de $\mathrm{Cl} a$ en células del microfitoplancton.

Los cambios estacionales de las variables biológicas en las bahías de Manzanillo son principalmente influenciados por los procesos dinámicos regionales que ocurren en el noreste del océano Pacífico tropical adyacente, en donde se tiene la presencia de agua de la Corriente de California en invierno-primavera y de la Corriente Costera Mexicana en verano-otoño. La formación de giros ciclónicos en la zona de transición tropical-subtropical durante invierno y primavera provocan afloramientos de agua de periodo variable que generan en ocasiones mareas rojas. Estos eventos producen un incremento en la concentración de clorofila en las aguas superficiales y disminución en la magnitud del coeficiente específico de absorción por fitoplancton debido al efecto de paquete y a la comunidad fitoplanctónica dominada por células del microfitoplancton.

\section{Agradecimientos}

Este trabajo fue financiado por el Fondo Ramón Álvarez Buylla de Aldana con número 224/03 y por el proyecto CB-2011-01-167361 del CONACYT. Asimismo, agradecemos a Julieta Hernández-López, Tania J. GonzálezAlejandre, Víctor M. Castro-Cabello, J.M. Domínguez, y F.J. Ponce por el apoyo en gabinete, campo y laboratorio. Agradecemos las sugerencias de los revisores anónimos que con sus comentarios mejoró significativamente la calidad del manuscrito.

\section{LITERATURA CITADA}

Anderson DM. 1995. ECOHAB: The ecology and oceanography of harmful algal blooms: A national research agenda, $66 \mathrm{pp}$. Woods Hole Oceanographic Institution, Woods Hole.

Babin M, D Stramski, GM Ferrari, H Claustre, A Bricaud, G Obolensky \& N Hoepffner. 2003. Variations in the light absorption coefficients of phytoplankton, nonalgal particles, and dissolved organic matter in coastal waters around Europe. Journal Geophysical Research 108: 3211 <doi: 10.1029/2001 JC000882>

Badán-Dangón A. 1997. La corriente costera de Costa Rica en el Pacífico Mexicano. En: Lavín MF (ed). Contribución a la oceanografía física en México, pp. 99-113. Unión Geofísica Mexicana, México.

Balech E. 1988. Los dinoflagelados del Atlántico Sudoccidental. Publicaciones Especiales. Instituto Español de Oceanografía 1: 1-219. Ministerio de Agricultura, Pesca y Alimentación, Madrid.

Barocio-León OA, R Millán-Núñez, E Santamaría-delÁngel, A González-Silvera, CC Trees \& E OrellanaCepeda. 2008. Bio-optical characteristics of a phytoplankton Bloom event off Baja California Peninsula $\left(30-31^{\circ} \mathrm{N}\right)$. Continental Shelf Research 28: 672-681.

Bricaud A, H Claustré, J Ras \& K Oubelkheir. 2004. Natural variability of phytoplankton absorption in oceanic waters: Influence of the size structure of algal populations. Journal of Geophysical Research 109, C11010: 1-12.

Castro-Ochoa FY. 2010. Identificación de grupos fitoplanctónicos a través de pigmentos trazadores en Bahía de Santiago, Colima durante primavera y verano de 2008 . Tesis de Licenciatura, Facultad de Ciencias Marinas, Universidad de Colima, Manzanillo, 46 pp.

Cleveland JS \& AD Wiedemann. 1993. Quantifying absorption by aquatic particles: A multiple scattering correction for glass fiber filters. Limnology and Oceanography 38(6): 1321-1327.

Cortés-Altamirano R \& F Páez-Osuna. 1998. Dinámica de las mareas rojas. En: Cortés-Altamirano R (ed). Las mareas rojas, pp. 1-12. AGT, México. 
Gárate-Lizárraga I. 2009. First record of Ceratium dens (Dinophyceae) in the Gulf of California. CICIMAR Oceánides 24(2): 167-173.

Godínez VM, E Beier, MF Lavín \& A Kurczyn. 2010. Circulation at the entrance of the Gulf of California from satellite altimeter and hydrographic observation. Journal of Geophysical Research 115, C04007<http://dx.doi.org/ 10.1029/2009JC005705>

IOCCG. 2000. Remote sensing of ocean colour in coastal, and other optically-complex, waters. In: Sathyendranath S (ed). Reports of the International Ocean-Colour Coordinating Group 3: 1-140. IOCCG, Dartmouth.

Kahru M \& BG Mitchell. 1998. Spectral reflectance and absorption of a massive red tide off southern California. Journal of Geophysical Research 103: 21601-21609.

Kim HG, SG Lee \& CS Jeong. 2004. Observations of coastal eutrophication and the succession of HAB species in Korean waters over three decades. A workshop on: Contrasting approaches to understanding eutrophication effects on phytoplankton. ICES Working Group on Phytoplankton Ecology (WGPE) den Haag, pp. 11-13.

Kishino M, N Takahashi, N Okami \& S Ichimura. 1985. Estimation of the spectral absorption coefficients of phytoplankton in the sea. Bulletin of Marine Science 37: 634-642.

Kurczyn JA, E Beier, MF Lavín \& A Chaigneau. 2012. Mesoscale eddies in the northeastern Pacific tropical subtropical transition zone: statistical characterization from satellite altimetry. Journal of Geophysical Research 117, C10021, <http://dx.doi.org/10.1029/2012JC007970>

Manrique FA \& RE Molina. 1997. Presencia de mareas rojas en la bahía de Bacochibampo, Guaymas, Sonora, México. Hidrobiológica 7: 81-86.

Meave del Castillo ME, ME Zamudio-Reséndiz, YB Okolodkov \& IH Salgado-Ugarte. 2003. Ceratium balechii sp. nov. (Dinophyceae: Gonyaulacales) del Pacífico Mexicano. Hidrobiológica 13(1): 75-91.

Megard RO \& T Berman. 1989. Effects of algae on the Secchi transparency of the southeastern Mediterranean Sea. Limnology and Oceanography 34: 1640-1655.

Millán-Núñez E, ME Sieracki, R Millán-Núñez, JR LaraLara, G Gaxiola-Castro \& CC Trees. 2004. Specific absorption coefficient and phytoplankton biomass in the southern region of the California Current. Deep-Sea Research II 51: 817-826.
Millán-Núñez E \& R Millán-Núñez. 2010. Specific absorption coefficient and phytoplankton community structure in the Southern Region of the California Current during January 2002. Journal of Oceanography 66: 719730 .

Parsons TR, Y Maita \& CM Lalli. 1984. A manual of chemical and biological methods for seawater analysis, $173 \mathrm{pp}$. Pergamon Press, New York.

Sathyendranath S, RP Bukata, R Arnone, MD Dowell, CO Davis, M Babin, J-F Berthon, OV Kopelevich \& JW Campbell. 2000. Colour of case 2 waters. In: Sathyendranath S (ed). Remote sensing of ocean colour in coastal, and other optically-complex, waters,. Reports of the International Ocean-Colour Coordinating Group 3: 2346. IOCCG, Dartmouth.

Sosa-Ávalos R, G Gaxiola-Castro, BG Mitchell \& J CepedaMorales. 2010. Coeficiente específico de absorción de luz por fitoplancton. En: Gaxiola-Castro G \& R Durazo (eds). Dinámica del ecosistema pelágico frente a Baja California, 1997-2007, pp. 333-347. Secretaría del Medio Ambiente y Recursos Naturales, Centro de Investigación Científica y de Educación Superior de Ensenada, Universidad Autónoma de Baja California, México.

Sosik H \& BG Mitchell. 1995. Light absorption by phytoplankton, photosynthetic pigments and detritus in the California Current System. Deep-Sea Research 42: 17171748.

Utermöhl H. 1958. Zur vervollkommnung der quantitativen phytoplankton-methodik. Mitteilung Internationale Vereinigung für Theoretiche und Angewandte Limnologie 9: 1-38.

Vargas-Montero M \& E Freer. 2004. Presencia de los dinoflagelados Ceratium dens, C. fusus y C. furca (Gonyaulacales: Ceratiaceae) en el Golfo de Nicoya, Costa Rica. Revista de Biología Tropical 52: 115-120.

Venrick EL \& TL Hayward. 1984. Determining chlorophyll on the 1984 CalCOFI surveys. California Cooperative Oceanic Fisheries Investigations Reports 25: 74-79.

Wu J, H Hong, S Shang, M Dai \& Z Lee. 2007. Variation of phytoplankton absorption coefficients in the northern South China Sea during spring and autumn. Biogeosciences Discuss 4: 1555-1584.

Zar JH. 1999. Biostatistical analysis, 663 pp. Prentice Hall, Englewood Cliffs.

Recibido el 25 de marzo de 2014 y aceptado el 10 de diciembre de 2014

Editor: Claudia Bustos D. 